\title{
Cosmic ray streaming instability generated in the intergalactic medium
}

\section{Marta D'Angelo*}

Gran Sasso Science Insitute-INFN

E-mail: marta.dangelo@gssi.infn.it

\section{Pasquale Blasi}

INAF/Osservatorio Astrofisico di Arcetri

Gran Sasso Science Insitute-INFN

E-mail: blasi@arcetri.astro.it

\section{Elena Amato}

INAF/Osservatorio Astrofisico di Arcetri

E-mail: amatodarcetri.astro.it

\begin{abstract}
We study the effects of the cosmic ray instabilities excited by the current associated with an inhomogeneous distribution of sources of extragalactic cosmic rays, with average density $n_{s}$. We find that under certain conditions, the resulting current can excite a non-resonant instability that forces particles with energy below some critical value to be confined within a distance $n_{s}^{-1 / 3}$ from the sources. This also causes the existence of a pervasive intergalactic magnetic field with a strength of order $\sim 0.01 \mathrm{nG}$.
\end{abstract}

The 34th International Cosmic Ray Conference,

30 July- 6 August, 2015

The Hague, The Netherlands

\footnotetext{
${ }^{*}$ Speaker.
} 


\section{Introduction}

At present there is still a lack of agreement on the nature of extragalactic sources that may accelerate ultra-high energy cosmic rays (UHECRs) up to $E \geq 10^{19} \mathrm{eV}$. Following the argument proposed in Ref. [1], it is possible to put some lower bounds on the source luminosity $L_{C R}$. Indeed, by requiring that, whatever the source is, it has to accelerate charged particles to energies $\sim 10^{20} \mathrm{eV}$, one gets a lower limit on the source luminosity [1], $L_{C R} \geq 1.6 \times 10^{45} Z^{-2} \beta\left(\frac{E}{10^{20} \mathrm{eV}}\right)^{2} \mathrm{erg} / \mathrm{s}$, where $Z$ is the atomic number and $\beta$ is the dimensionless velocity of the accelerator in units of the speed of light $c$. In case of relativistic sources the limit becomes $L_{C R} \geq 10^{47} Z^{-2} \Gamma^{2}\left(\frac{E}{10^{20} \mathrm{eV}}\right)^{2} \mathrm{erg} / \mathrm{s}$ where $\Gamma$ is the Lorentz factor. These lower limits indicate that extragalactic sources have to be very luminous in order to accelerate UHECRs.

The diffuse flux of UHECRs produced by the whole source distribution depends on the product of $L_{C R}$ and on the space density of sources $n_{s}$. Therefore, by measuring comic ray (CR) spectra, it is possible to estimate for example $L_{C R}$ as a function of $n_{s}$ and even estimating $L_{C R}$ if additional information (such as small scale anisotropies) is available (see for instance [2]). Currently number densities of the order of $n_{s} \sim 10^{-5} \mathrm{Mpc}^{-3}$ (with a large uncertainty) are considered viable. The total luminosity of the source depends rather drastically on whether the injection spectrum assumed at the highest energies is extrapolated to low energies as well.

Even a source distribution which is perfectly homogeneous on average has fluctuations in the local number of sources, as a result of Poisson noise in the spatial distribution. Such fluctuations result in random dipole anisotropies in the arrival direction of CRs at any location in space, and these anisotropies translate into an effective CR electric current pervading the intergalactic medium (IGM), though in general the orientation of such current is random at different locations. Since the local electric charge and total current must vanish, compensation currents (motion of electrons) are induced in the background plasma, which may lead to plasma instabilities.

In this work we are interested in finding what are the conditions for the development of the non-resonant modes of the streaming instability initially discussed in a different context [3]. To achieve this goal, we first provide an estimate of the source anisotropy in $\$ 2$ for typical values of the source density, then we discuss the implications of the existence of the unstable modes for CR propagation and generation of an intergalactic magnetic field (§3). We conclude in $\S 4$.

\section{An estimate of CR anisotropy}

Even in the presence of a homogeneous distribution of sources in the Universe, Poisson fluctuations will induce a fluctuation in the number of sources in two opposite regions of the universe, thereby inducing a dipole anisotropy in the flux of cosmic rays $\delta=\Delta N_{s} / N_{s}$, where $N_{s}$ is the number of sources within one loss length at the given energy $E$ of CRs. For simplicity we assume here that all CRs are protons, so that the loss length is determined by adiabatic energy losses (at low energy), Bethe-Heitler pair production at energies above the threshold for this process, and photopion production on the photons of the cosmic microwave background (CMB) above $\sim 10^{20} \mathrm{eV}$. We are interested in studying the propagation over cosmological distances of particles with energy $<10^{9} \mathrm{GeV}$. Their energy loss length $\lambda_{\text {loss }}$ is a constant, as shown in Fig. 1 where $\lambda_{\text {loss }}$ is plotted as a function of particle energy. Indeed, in this range of energies the process which dominates is the 


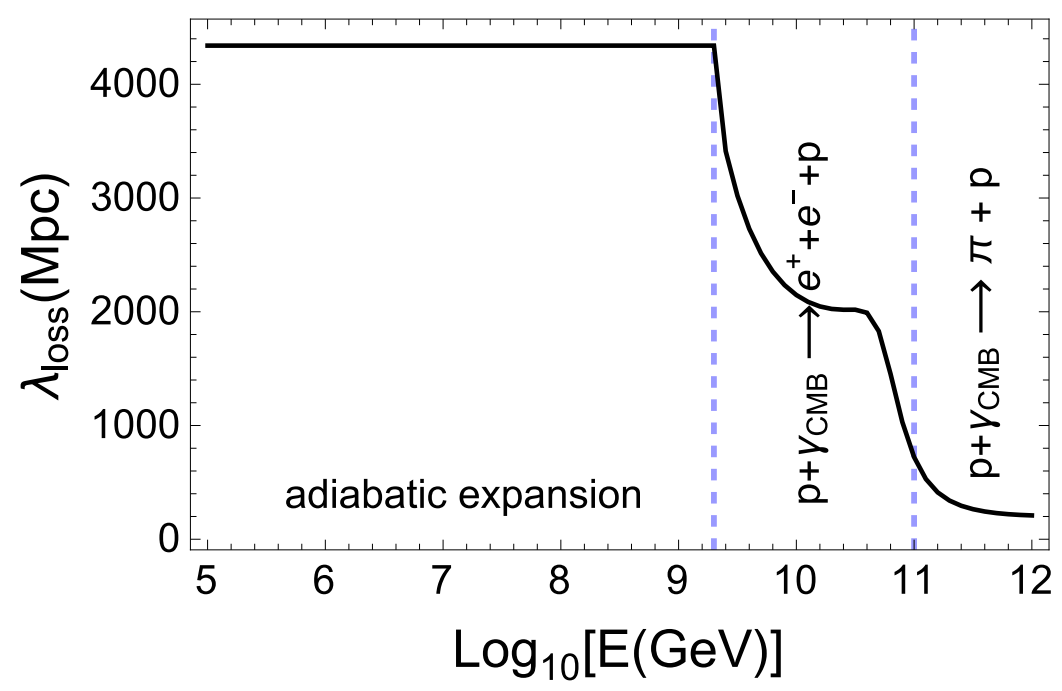

Figure 1: Energy loss length $\lambda_{\text {loss }}$ as a function of the logarithm of particle energy.

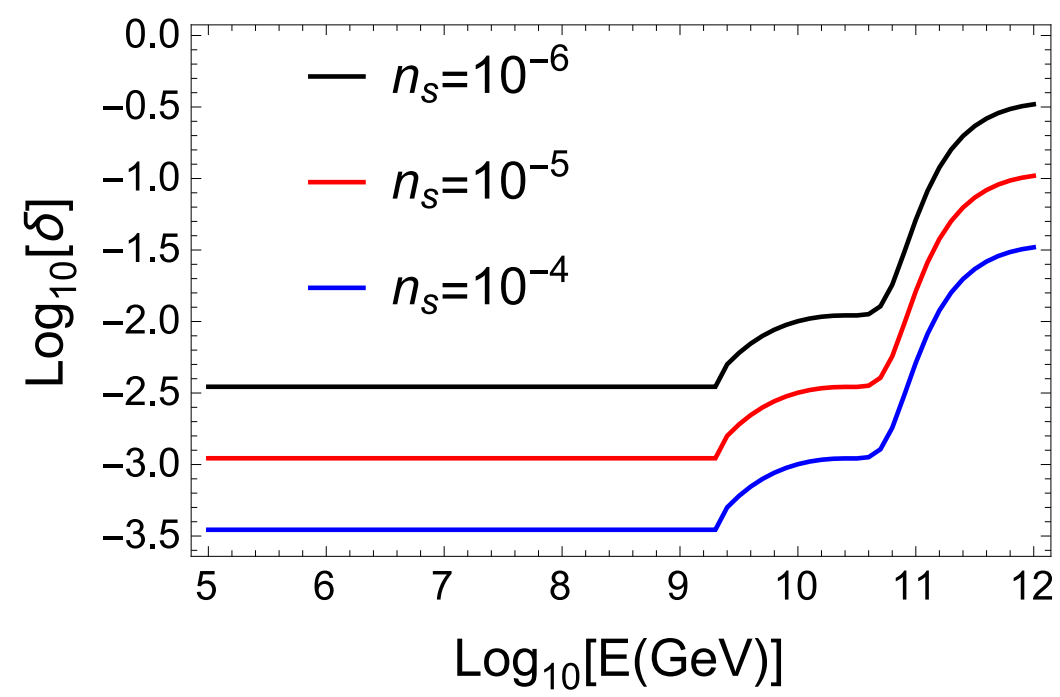

Figure 2: Plot of the logarithm of $\delta$ as a function of the logarithm of particle energy for three different values of $n_{s}: 10^{-6} \mathrm{Mcp}^{-3}$ black line, $10^{-5} \mathrm{Mcp}^{-3}$ red line and $10^{-4} \mathrm{Mcp}^{-3}$ blue line.

adiabatic expansion of the Universe, which means $\lambda_{\text {loss }}=c \tau_{H}=4.34 \times 10^{3} \mathrm{Mpc}$ (where $c$ is the speed of light and $\tau_{H}=1.4 \times 10^{10} \mathrm{yr}$ is the Hubble time). The number of sources which contribute to the CR dipolar anisotropy are those contained into the volume $V_{\text {loss }}=\lambda_{\text {loss }}^{3}$.

We assume that these extragalactic sources follows a Poisson distribution. Therefore, the estimation of the fluctuations is $\Delta N_{s}=\sqrt{N_{s}}$. Then the expression for the anisotropy becomes

$$
\delta=\frac{\Delta N_{s}}{N_{s}}=\frac{1}{\sqrt{V_{\text {loss }} n_{s}}} .
$$

We plot the profile of $\log _{10} \delta(E)$ in Fig. 2 for three different values of $n_{s}$. For $E<10^{9} \mathrm{GeV}$ the value of $\delta$ is of the order of $10^{-3}$ in case the number density is $\sim 10^{-5} \mathrm{Mpc}^{-3}$. 


\section{Conditions for CR non-resonant streaming instability}

As pointed out in $\S 1$ even an homogeneous distribution of sources induces a dipolar anisotropy in the CR distribution, due to Poisson fluctuations in the number of such sources. Consequently a CR electric current $J_{C R}$ propagates into the IGM. The system made of this electric current and the magnetized plasma of the IGM can be subject to a host of instabilities. The instability which grows faster than all the others is the non-resonant streaming instability (also called Bell's instability, see Ref. [3]). The unstable modes induce an important amplification of the magnetic turbulence which leads to an enhancement of particle's scattering, which in turn leads to diffusive particle transport. This phenomenon also occurs in the immediate proximity of the source, as discussed in Ref. [4]. We estimate the CR spatial density $n_{C R}$ by the diffusive flux measured on Earth (see for example Fig. 3 reported in Ref. [5]), obtaining $n_{C R} \sim 4.8 \times 10^{-8}\left(E_{\mathrm{GeV}}\right)^{-2} \mathrm{~cm}^{-3}$. If the minimum energy of particles in the current is $E, J_{C R}$ can be written as $e n_{C R}(E) v_{d}$, where the drift velocity is $v_{d}=\delta c$.

We assume that the baryonic component of the IGM has a density $n_{b} \simeq 2.5 \times 10^{-7} \mathrm{~cm}^{-3}$ and that there is a cosmological magnetic field with a strength $B_{0}$, even if there are only very low bound limits on it. The corresponding Alfvén speed is $v_{A}=B_{0} / \sqrt{4 \pi m_{p} n_{b}} \approx 44 B_{0} /\left(10^{-13} \mathrm{G}\right) \mathrm{cm} / \mathrm{s}$.

A first condition to satisfy for the growth of these modes is that the energy density of CRs has to be locally larger than the energy density of the cosmological magnetic field, i.e. $J_{C R} E / c e>B_{0}^{2} / 4 \pi$ ( $e$ indicates the proton charge). This condition can be expressed as

$$
\delta \frac{n_{C R}(E)}{n_{b}}\left(\frac{c}{v_{A}}\right)^{2} \frac{E}{m_{p} c^{2}}>1 .
$$

Eq. (3.1) imposes a limit on particle energy. The system is in the CR current driven regime for those particles which have their energy less than a critical value $E_{c 1}$ :

$$
E<E_{c 1}=9.1 \times 10^{16} \delta\left(\frac{B_{0}}{10^{-13} \mathrm{G}}\right)^{-2} \mathrm{GeV} .
$$

In the range of energy we are interested in, $E<10^{9} \mathrm{GeV}, \delta \sim 10^{-3}$ (see Fig. 2) which means $E_{c 1} \geq 10^{13} \mathrm{GeV}$. Therefore, the first condition is satisfied.

There is a more restrictive limit that comes from the fact that the non-resonant modes are required to grow on time scales not longer than the age of Universe to be relevant. The growth rate of the fastest growing mode is

$$
\gamma_{\text {max }}=k_{\text {max }} v_{A}=\sqrt{\frac{4 \pi}{n_{p} m_{p}}} e n_{C R}(E) \delta,
$$

where $k_{\max }$ is obtained by balancing the magnetic tension with the $J_{C R} \times \delta B$ force, as explained in Ref. [3]. Comparing $\gamma_{\max }^{-1}$ with $\tau_{H}$ we obtain a second critical energy $E_{c 2}$ :

$$
E_{c 2}=2.4 \times 10^{8} \delta^{1 / 2} \mathrm{GeV} \text {. }
$$

Only for those particles with $E<E_{c 2}$ the non-resonant modes are able to grow. We rewrite $E_{c 2}$ as a function of $n_{s}$.Thus, eq. (3.4) reads

$$
E<E_{c 2}=4.45 \times 10^{5}\left(n_{s}\right)^{-1 / 4} \mathrm{GeV} .
$$


The first effect we want to study is the amplification of the magnetic turbulence $B$. We assume that the saturation regime is reached when the Larmor radius in the amplified field is bigger or of the same order of the length scale over which the magnetic field grows, i.e. $r_{L}(B) \sim 1 /(\xi k)$, where the parameter $\xi$ goes from 0 to 1 corresponding to the upper limit case $\left(r_{L}(B) \sim 1 / k\right)$. The length scale $k$ is estimated by the comparison between the magnetic tension $B_{0} \times B k / 2 \pi$ and the $J_{C R} \times B / c$ force. Therefore, substituting $k$ into the first condition we obtain the following expression for the amplified magnetic turbulence:

$$
B=\xi \sqrt{4 \pi n_{C R}(E) E \delta} .
$$

We note that this expression is independent of the background magnetic field $B_{0}$. With this value of $B$ it is possible to estimate the effect on particle propagation over cosmological distances. As mentioned before, there is an enhancement of particle's scattering which leads to a diffusive propagation of cosmic rays. In order to derive an estimate of $D$ we assume Bohm diffusion in the self-generated turbulence, that is (UHECRs are ultra-relativistic so their velocity is $c$ )

$$
D=\frac{1}{3} r_{L}(B) c=5.73 \times 10^{32} \xi^{-1}\left(n_{s}\right)^{1 / 4}\left(\frac{E}{10^{6} \mathrm{GeV}}\right)^{3 / 2} \mathrm{~cm}^{2} \mathrm{~s}^{-1} .
$$

We consider the diffusion of particles over a length scale $d$ corresponding to the mean distance between two sources, which is of the order of $\sim\left(n_{s}\right)^{-1 / 3}$. The particles for which the diffusion time $\tau_{d}$ is shorter than the age of the Universe are able to propagate away from sources and are the particles which contribute effectively to the CR electric current. Therefore, we calculate $\tau_{d}$ and we compare it to $\tau_{H}$. The diffusion time is estimated as $d^{2} / D$, which reads:

$$
\tau_{d}=5.24 \times 10^{8} \xi\left(n_{s}\right)^{-11 / 12}\left(\frac{E}{10^{6} \mathrm{GeV}}\right)^{-3 / 2} \mathrm{yr} .
$$

The comparison between $\tau_{d}$ and $\tau_{H}$ imposes a third condition on particle energy

$$
E<E_{c 3}=1.12 \times 10^{5} \xi^{2 / 3}\left(n_{s}\right)^{-11 / 18} \mathrm{GeV} .
$$

When eq. (3.5) and eq. (3.9) are satisfied in such a way that $E_{c 3}<E_{c 2}$ particles produce nonresonant modes and the self-generated magnetic turbulence forbids their propagation over distances larger than $\left(n_{s}\right)^{-1 / 3}$. Fig. 3 shows $E_{c 2}$ and $E_{c 3}$ as a function of $n_{s}$ for three different values of $\xi$. We observe that the constraint $E_{c 3}<E_{c 2}$ is satisfied only for a certain range of $n_{s}$ which changes with $\xi$. In this range, the particles with $E>E_{c 3} \sim 10^{7} \mathrm{GeV}$ contribute effectively to the electric current $J_{C R}$ because lower energy particles are self-confined within a distance $n_{s}^{-1 / 3}$ from the sources.

We can estimate the value of the magnetic field at saturation which corresponds to the expression reported in eq. (3.6) calculated at $E=E_{c 3}$, that is

$$
B_{\text {sat }} \approx 1.01 \times 10^{-11}\left(\frac{n_{s}}{10^{-4.5} \mathrm{Mpc}^{-3}}\right)^{1 / 18}\left(\frac{\xi}{1 / 30}\right)^{2 / 3} \mathrm{G} .
$$

The length scale over which the saturation is established is $r_{L}\left(B_{\text {sat }}\right) \sim 10^{7} /\left(e B_{\text {sat }}\right) \sim 1 \mathrm{Mpc}$.

In the numerical simulations of Bell's instability performed by Riqueleme and Spitkovsky [6], the saturation regime is reached when the following condition is fulfilled:

$$
k_{\text {num }} r_{L}(B)=1 \quad \text { with } \quad k_{\text {num }}=2 k\left[\left(\frac{B_{0}}{B}\right)^{2}+1\right]^{-1}
$$






Figure 3: Plot of the two critical values $E_{c 2}$ and $E_{c 3}$ as a function of $n_{s}$ for three different values of $\xi$ : $(a) 1$, (b) $1 / 10$ and $(c) 1 / 30$.

where $k$ is the wavenumber of the amplified field when the magnetic tension is of the same order of $J_{C R} \times B / c$ force. The expression for the turbulence is

$$
B_{\text {num }}=B(\xi=1)\left(18 \frac{B_{0}}{B(\xi=1)}\right)^{1 / 3}
$$

where we have used the subscript ${ }_{\text {num }}$ to distinguish the value obtained using numerical simulations with respect to the theoretical one, $B$, reported in eq. (3.6). We note that the value of $B_{\text {num }}$ depends on the cosmological magnetic field $B_{0}$. As before, we estimate the diffusion coefficient assuming Bohm diffusion

$$
D_{\text {nит }}=1.83 \times 10^{33}\left(\frac{E}{10^{6} \mathrm{GeV}}\right)^{4 / 3}\left(n_{s}\right)^{1 / 6}\left(\frac{B_{0}}{10^{-13} \mathrm{G}}\right)^{-1 / 3} \mathrm{~cm}^{2} \mathrm{~s}^{-1} .
$$

Then we calculate the diffusion time over a mean distance between two sources $\sim\left(n_{s}\right)^{-1 / 3}$, that is

$$
\tau_{d}^{\text {num }}=1.65 \times 10^{8}\left(\frac{E}{10^{6} \mathrm{GeV}}\right)^{-4 / 3}\left(n_{s}\right)^{-5 / 6}\left(\frac{B_{0}}{10^{-13} \mathrm{G}}\right)^{1 / 3} \mathrm{yr} .
$$

The comparison between the diffusion time and the age of the Universe gives a critical energy

$$
E_{c 3}^{\text {num }}=3.57 \times 10^{4}\left(n_{s}\right)^{-5 / 8}\left(\frac{B_{0}}{10^{-13} \mathrm{G}}\right)^{1 / 4} \mathrm{GeV} .
$$

The condition $E_{c 3}<E_{c 2}$ is satisfied when $n_{s}>10^{-3} \mathrm{Mpc}^{-3}$ and the magnetic field at saturation is

$$
B_{\text {sat }}^{\text {num }}=4.15 \times 10^{-11}\left(\frac{n_{s}}{10^{-3} \mathrm{Mpc}^{-3}}\right)^{1 / 24}\left(\frac{B_{0}}{10^{-13} \mathrm{G}}\right)^{1 / 4} \mathrm{G} .
$$




\section{Conclusions}

We consider a distribution of the extragalactic sources that is constant in space and time. Nevertheless, there are statistical fluctuations in the number of the sources which induce a dipolar anisotropy $\delta$ in the CR distribution. For the energies we are interested in, that is $E<10^{9} \mathrm{GeV}$, $\delta \sim 10^{-3}$ and depends only on the number density $n_{s}$. An anisotropic CR distribution generates an electric current which propagates into the IGM and induces various plasma instabilities. The fastest is the non-resonant streaming instability (also called Bell's instability, see ref. [3]). There are two important effects: the generation of a magnetic turbulence and a self-confinement mechanism for particles with energy less than a critical value.

We estimated that the self-generated magnetic field is $\sim 10^{-11} \mathrm{G}$ over a length scale of the order of $1 \mathrm{Mpc}$.

We have observed that particles with $E>10^{7} \mathrm{GeV}$ are able to propagate over cosmological distances. Therefore, they contribute effectively to the CR electric current which induces the nonresonant streaming instability. The self-generated magnetic turbulence enhances particle's scattering and consequently reduces the diffusion coefficient in such a way that particles with $E<10^{7} \mathrm{GeV}$ remain confined over a distance comparable to the mean distance between two sources $\left(\sim n_{s}^{-1 / 3}\right)$.

\section{References}

[1] E. Waxman, Extra galactic sources of high energy neutrinos, Physica Scripta 2005 (2005), no. T121 147.

[2] P. Blasi and D. D. Marco, The small scale anisotropies, the spectrum and the sources of ultra-high energy cosmic rays, Astroparticle Physics 20 (2004), no. 5559 - 577.

[3] A. R. Bell, Turbulent amplification of magnetic field and diffusive shock acceleration of cosmic rays, Monthly Notices of the Royal Astronomical Society 353 (sep, 2004) 550-558.

[4] P. Blasi, E. Amato, and M. D'Angelo, High energy cosmic ray self-confinement close to extragalactic sources, submitted to PRL 2015 (2015).

[5] R. Aloisio, V. Berezinsky, P. Blasi, A. Gazizov, S. Grigorieva, and B. Hnatyk, A dip in the \{UHECR\} spectrum and the transition from galactic to extragalactic cosmic rays, Astroparticle Physics 27 (2007), no. $176-91$.

[6] M. A. Riquelme and A. Spitkovsky, Nonlinear study of bell's cosmic ray current-driven instability, The Astrophysical Journal 694 (2009), no. 1626. 